\title{
Activity based models for countrywide electric vehicle power demand calculation
}

\author{
Luk Knapen*, Bruno Kochan*, Tom Bellemans*, Davy Janssens* and Geert Wets* \\ *Transportation Research Institute (IMOB) \\ Hasselt University, Wetenschapspark 5 bus 6, 3590 Diepenbeek, Belgium \\ Email: geert.wets@uhasselt.be
}

\begin{abstract}
Smart grid design depends on the availability of realistic data. In the near future, energy demand by electric vehicles will be a substantial component of the overall demand and peaks of required power could become critical in some regions. Transportation research has been using micro-simulation based activity-based models for traffic forecasting. The resulting trip length distribution allows to estimate to what extent internal combustion engine vehicles can be substituted by electric vehicles. Second, combining the results emerging from activity based models with assumptions on electric vehicles market share, allows to predict energy and power demand in time and space. Furthermore, smart grid management effects can be investigated using activity based models because generated schedules determine how charging periods can float in time. This paper presents results calculated for the Flanders region.
\end{abstract}

\section{INTRODUCTION}

Activity based models (AB models) are behavioral models predicting activity schedules which can be used to derive transportation needs. Model parameters are calculated from census demographic data and from periodic dedicated mobility surveys (Onderzoek Verplaatsingsgedrag, OVG). A synthetic population is generated and an activity schedule is generated for each household member on a typical working day. The model output consists of predicted activities and trips for which origin, destination, motive, time-of-day, transportation mode, household characteristics and more attributes are known. $\mathrm{AB}$ models are based on microsimulation of individual actors. Because of the rich information content, results can be used for various types of analysis [1].

Several scenarios of electric vehicle (EV) market penetration have been studied. First we explain what hypotheses about EV drivers behavior have been made and how EV characteristics have been determined from literature and from available statistics. Next some details of the calculation are described. Finally, results for the Flanders region (6 million inhabitants, 13000 square kilometers) in Belgium (11 million inhabitants, 30000 square kilometers) are presented.

\section{CONTEXT}

\section{A. Energy vs. Power}

According to several sources ([2],[3]) the total amount of energy drawn from the grid by electric vehicles is relatively small : a $30 \%$ market share EV would represent $3 \%$ of the total annual electric energy consumption for the region of Milan, Italy.
The amount of energy required yearly for a household EV is of the same order of magnitude as the current household electric energy consumption. According to figures published in Oxford University Environmental Change Institute website statistics pages ([4]) the average yearly consumption for a belgian household amounts to 3899 [kWh/year]. A similar figure (3500 [kWh/year]) for Belgium is mentioned by [5]. Power demand while charging electric vehicles however deserves attention since charging time and location highly influence the size of peak demand. Perujo and Ciuffo [3] studied power demand for the Milan region using the assumptions that people will not charge their car batteries everyday but only when needed and that charging starts between 16:00h and 19:00h in the evening obeying a uniform distribution over time. Those assumptions can be refined : [2] recognizes the need for statistics on daily commuter trips for a particular region. The study described in this article refines the assumptions about charging time and location by using the results of an activity based transport demand model.

\section{B. The use of activity based models}

In order to dimension the electric grid parameters, accurate estimates for power demand as a function of time and location are essential. Such estimates can be based on the results of calibrated activity based (AB) transport demand models because those models deliver detailed location, timing and motivation information about trips and activities for each individual. Two strategies for battery charging have been used to calculate peak power demand as a function of time and location :

- Scenario 1 : people start charging as soon as possible during the low tariff period. This scenario is used as a reference.

- Scenario 2 : people start charging at a uniformly distributed moment in time and so that their cost is minimal (maximum use of low tariff period) which looks like a decentralised smartgrid strategy for peak shaving.

In both cases, charging period is assumed to be contiguous (uninterrupted). Furthermore we hypothesize that everyone will recharge batteries everyday due to the well known range anxiety. This study has been limited to two scenarios. However, since AB models are microsimulation based, each individual actor behaves in its own specific way. Different 


\begin{tabular}{|l|r|r|r|}
\hline & \multicolumn{3}{|c|}{ Motor cylinder volume } \\
\hline & $V<1400$ & $1400 \leq V \leq 2000$ & $2000<V$ \\
\hline EV category & small & medium & large \\
\hline Market share & 0.496 & 0.364 & 0.140 \\
\hline $\begin{array}{l}\text { Battery capacity } \\
{[\mathrm{kWh}]}\end{array}$ & 10 & 20 & 35 \\
\hline Range $[\mathrm{km}]$ & 100 & 130 & 180 \\
\hline $\begin{array}{l}\text { Energy consump- } \\
\text { tion }[\mathrm{kWh} / \mathrm{km}] \\
: \text { lower limit }\end{array}$ & 0.095 & 0.138 & 0.175 \\
\hline $\begin{array}{l}\text { Energy consump- } \\
\text { tion }[\mathrm{kWh} / \mathrm{km}] \\
: \text { upper limit }\end{array}$ & 0.110 & & \\
\hline
\end{tabular}

TABLE I

TECHNICAL CHARACTERISTICS FOR VEHICLES IN SPECIFIC CATEGORIES. TO DETERMINE MARKET SHARE, EV CATEGORIES ARE MAPPED TO ICE CATEGORIES FOR WHICH MARKET SHARE IS KNOWN.

actor categories thus can show different preferences and particular scenarios based on specific actor classifications can be evaluated.

\section{Related work}

Clement-Nyns et al. [6] evaluate coordinated charging strategies for a belgian case. In such systems customers need to specify time limits for charging (which can be produced by AB-models). Waraich et al. [7] evaluate energy tariff effects on charging behavior for the city of Berlin by coupling MATSim-T (travel demand simulator framework) to PMPSS (PHEV Management and Power Systems Simulation).

\section{GRID LOAD CALCULATION}

Since the EV market is only emerging, predictions cannot be based on extensive statistics. The assumptions made have been explained and argued below.

\section{A. Vehicle categories}

Electric cars are subdivided into the categories small, medium, large similar to what is done in [3]. In order to estimate the energy requirement, one needs to know the contribution of each one of those categories to the complete vehicle set. Belgian government statistics provide a classification of internal combustion engine (ICE) vehicles based on the motor cylinder volume : they provide a distribution of the registered cars using that classification. We state the one-to-one mapping of categories given in table I that shows market share and technical characteristics for each category. Vehicle characteristics in the table have been derived from data in [3] and [8], the market share figures have been taken from the belgian federal government 2009 statistics (PARC010 Transport Indicator) [9]

\section{B. Available Chargers}

Two types of chargers are considered : $3.3[\mathrm{kVA}]$ and $7.2[\mathrm{kVA}]$ chargers compatible with the flemish grid. Our model distinguishes between home and work location chargers. Charger type occurrence probability is given in table II. The power value for home chargers is assumed to depend on the car category : smaller cars are equipped with a less powerfull charger. On the other hand, companies offering car charging facilities provide powerful chargers in order to save time and extend the distance that can be bridged during one day. Furthermore, electric energy is hypothesized to be less expensive than fuel (mainly due to tax reasons).

\section{Company cars in Belgium}

Employers are believed to allow company car (CC) drivers to charge at the work location because that is less expensive than providing fuel cards to employees. However, for technical reasons, some companies cannot provide the required infrastructure. The fraction of actors who can charge batteries at the work location has been determined as a fraction of company car drivers. It has been assumed that $50 \%$ (FRAC_WORK_CHARGERS in table III) of the work locations provides suitable infrastructure for battery charging.

The AB model predicts trips and provides information about car availability but not about car ownership. In order to estimate the number of people able to charge batteries at the work location, we need to estimate the fraction of work trips traveled by company car.

The number of company car users has been derived from following sources. Gutiérrez [10] page 2 cites a report stating that in Belgium $20 \%$ of the employees have a company car. According to [11] (survey conducted by SD-WORX) $28.9 \%$ of the belgian employees had a company car available in 2007. Many reports use company car ownership as an independent variable to classify quantities like average trip distance, willingness to change to public transport ...but do not mention company car market share figures. On the other hand, the COCA (Company Car analysis) report [12] states that depending on the context, multiple definitions of a company car (voiture de société) are in use because both fiscal and operational aspects are concerned. The COCA definition (A company car is made available by a company to an employee for both professional and private use) is used in our study. The report only states that $45 \%$ of new car registrations in Belgium are for company cars but does not mention the fraction of employees having a company car at their disposal. The same COCA report states that, based on two belgian reports ( $O V G$ for Flanders and $E R M M W$ for Wallonia), it can be concluded that $6 \% \ldots 7 \%$ of the car fleet in use by belgian households, is company owned (source [12] page $31 / 80$ ). Another method described in [12] page 31-80 estimates that $10 \%$ of all registered cars in Belgium are company cars. Therefore, this report assumes for the model that $10 \%$ of the actors driving to work, make use of a company car.

\section{Method overview}

The Feathers software [13] created by the Transportation Research Institute (IMOB) has been used to generate activitytravel schedules (daily agenda for each individual of the flemish population). Each schedule consists of trip and activities. For each trip, departure time, trip duration, origin and destination zones are predicted. For each activity, the purpose (work, shop, bring-get, ...) is predicted. In this study, only 


\begin{tabular}{|l|r|r|}
\hline Car category & Prob(3.3[kW]) & Prob(7.2) $[\mathrm{kW}]$ \\
\hline \multicolumn{3}{|c|}{ Home } \\
\hline small & 0.8 & 0.2 \\
medium & 0.4 & 0.6 \\
large & 0.1 & 0.9 \\
\hline \multicolumn{2}{|c|}{ Work } \\
\hline small & 0.1 & 0.9 \\
medium & 0.1 & 0.9 \\
large & 0.1 & 0.9 \\
\hline
\end{tabular}

TABLE II

CHARGER TYPE DISTRIBUTION

\begin{tabular}{|l|l|r|}
\hline DCD_FRAC & Deep charge depletion fraction & 0.1 \\
off2realCorrection & Range reduction factor & 0.85 \\
CC_PROBABILITY & Fraction of employees driving a & 0.1 \\
& $\begin{array}{l}\text { company car } \\
\text { FRAC_WORK_CHARGERS }\end{array}$ & $\begin{array}{l}\text { Fract of CC drivers charging at } \\
\text { work location }\end{array}$ \\
lowTariffFirst & Low tariff period first minute & 0.5 \\
lowTariffLast & Low tariff period last minute & 22:00h \\
\hline
\end{tabular}

TABLE III

SIMULATION PARAMETERS

work and non-work activities are distinguished. Feathers results apply to a single 24-hour period. A working day simulation has been used in this study.

An application has been written to compute energy and power demand from Feathers results in 3 steps as follows :

- In a first step, schedules having at least one car trip are extracted and data structures are set up.

- In the second step, car ownership, possibility of work location charging, car characteristics (range, distance specific energy consumption, battery capacity) and the types of home and work location charger used, are determined. In this way, an electric car is assigned to each schedule in order to calculate the feasibility of electrification. Finally, a feasibility indicator is calculated : it tells whether or not the schedule can be executed using the assigned electric car. Each schedule is assumed to be executed using a single car and a predefined fraction of the company cars can get recharged at the work location as explained in section III-C; for details, refer to section III-G. The set of electrically feasible schedules is used for stochastic sampling during the third step. Feasible schedules are kept as a population from which to draw items to implement different electrification scenarios. The set of electrically feasible schedules is partitioned as specified in diagram 1. For each one of the leaf node parts, the market share has been specified : the results shown in this report hold for $10 \%$ no-work trip and 5\% work trip electrification.

- In the third step, charging scenarios are evaluated. Schedules are sampled from the partitions set up in the second step and the start time for each charging operation is determined. Energy requirement and power demand are accumulated for every minute of the day for each zone.

All parameters mentioned below have been smmarized in table III.

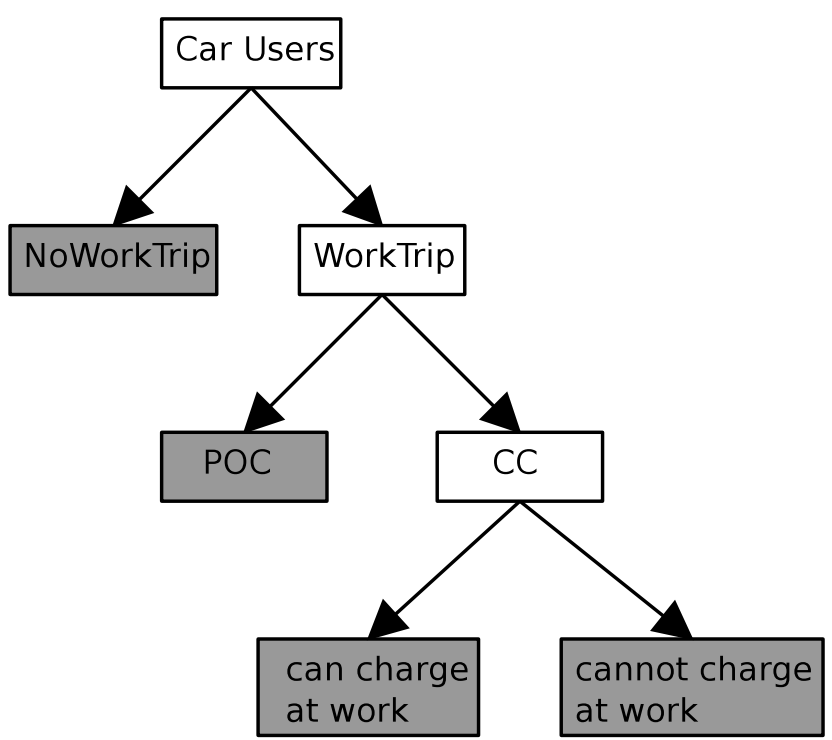

Fig. 1. Car users partitioning : market share is specified for shaded subsets

\section{E. Vehicle ownership}

Cars not used for any work trips are assumed to be privately owned cars. From the cars used for work trips, a fraction is taken to be a company car according to the data given in section III-C.

\section{F. Vehicle characteristics determination}

Vehicle characteristics are determined by random selection, independently of schedule characteristics.

- Vehicle category is randomly selected from the distribution specified in table I

- The probability for work location charging (configuration setting) and the type of charger are determined (from table II).

- Vehicle range is selected from table I.

- Vehicle consumption is randomly selected using a uniform distribution in the interval specified for the vehicle category (from table I). This is the consumption determined by official US and European standard (FTP,WP.29, etc) test suites that do not account for cabin clima (heating, airco) nor for frequent acceleration and deceleration.

- The battery capacity is derived from range and distance specific consumption and has been verified with data found in literature ([8],[14],[15]).

- The specific energy consumption as determined by european standard methods (UNECE WP.29 R101) is argued to be an underestimation. The standardised test conditions differ from operating conditions : hence, a range reduction coefficient (off 2 realCorrection in table III) of 0.85 has been applied. After battery capacity calculation, the range reduction coefficient is used to adjust the specific consumption (which is used in schedule feasibility and energy demand calculations).

- Finally, the charger power is randomly selected for both home and work location chargers using the distribution 
specified in table II.

\section{G. Electrified schedules}

In order to be feasible, each location in the schedule shall be reachable when starting with a fully charged battery in the morning. A schedule shall fulfil the condition

$$
\forall i: C_{b}+\sum_{j=0}^{j<i} c_{d, j} * c_{p, j}-d_{O, i} * \text { con } \geq C_{b} * D C D
$$

where $i$ and $j$ are location indices, $C_{b}$ is the battery capacity, $D$ is the set of all destinations, $c_{d, i}$ is the charge-period duration at the i-th location and $c_{p, i}$ is corresponding power, $d_{O, i}$ is the total distance from the first origin to the i-th destination, cons is the distance specific energy consumption and $D C D$ is the maximal deep charge depletion.

- A deep charge depletion coefficient (DCD_FRAC table III) of 0.1 has been applied to specify the minimal battery level that shall be available at all times. It is used to model range anxiety and is used in electrification feasibility calculation.

\section{H. Charging parameters - Scenarios}

\section{1) Assumptions valid for all scenarios concerned:}

- Energy cost is assumed to conform to the current tariff scheme used in Belgium : it consists of one contiguous regular tariff period and one contiguous low tariff period during the night (from 22:00h to 06:00h) (see table III).

- The schedules apply to a working day and schedules are assumed to repeat on successive days. This assumption allows to determine the period of time available for recharging overnight. Everyone charging at home is assumed

- to recharge batteries everyday

- to minimise energy cost by charging during the low tariff period as much as possible

- Charging occurs during a single uninterrupted period of time.

For each schedule and each charging opportunity, the required charge duration for full recharge and the available charge period are calculated. The available charge periode is determined from the arrival and departure times at the charge location. If the available period length is larger than the required charge duration, their difference is the slack time (otherwise slack time equals zero). A non-zero slack time implies a degree of freedom for selecting the time to start charging. In many cases, there is an interval $\Delta t=\left[t_{0}, t_{1}\right]$ of starting times $t_{s}$ such that $\forall t_{s} \in \Delta t$ the energy cost is the same. The charge period either overlaps completely or not at all with the low tariff period.

2) Scenario specific assumptions:

- Scenario 1 : If the interval $\Delta t$ is contained in the low tariff period, the actor starts charging as soon as possible; otherwise, the actor starts charging as late as possible thereby pushing energy demand to the morning hours.

- Scenario 2 : The charge period start time $t_{s}$ is chosen from $\Delta t$ by random selection using a uniform distribution.

\begin{tabular}{|l|r|}
\hline nActorsWorking / nActors & 0.406 \\
nCarUsers / nActors & 0.555 \\
\hline nCarUsersWorking / nCarUsers & 0.531 \\
\hline avgTripsPerActor & 3.670 \\
avgWorkRelatedCarTripDistance [km] & 19.376 \\
\hline nTrips[work] / nTripsAll & 0.160 \\
nTrips[atHome] / nTripsAll & 0.403 \\
nTrips[bringGet] / nTripsAll & 0.091 \\
nTrips[shoppingOne] / nTripsAll & 0.103 \\
nTrips[social] / nTripsAll & 0.054 \\
nTrips[services] / nTripsAll & 0.056 \\
nTrips[touring] / nTripsAll & 0.029 \\
nTrips[leisure] / nTripsAll & 0.044 \\
nTrips[other] / nTripsAll & 0.059 \\
\hline
\end{tabular}

TABLE IV

FEATHERS RESULTS STATISTICS

\begin{tabular}{|l|l|}
\hline Partition & $\begin{array}{l}\text { Fraction of the } \\
\text { car using sched- } \\
\text { ules }\end{array}$ \\
\hline $\begin{array}{l}\text { Electrically feasible schedules without work trips } \\
\text { POC }\end{array}$ & 0.377 \\
\hline Electrically feasible schedules with work trips POC & 0.374 \\
\hline $\begin{array}{l}\text { Electrically feasible schedules with work trips CC, } \\
\text { no chargeAtWork }\end{array}$ & 0.021 \\
\hline $\begin{array}{l}\text { Electrically feasible schedules with work trips CC, } \\
\text { chargeAtWork }\end{array}$ & 0.024 \\
\hline Infeasible & 0.204 \\
\hline
\end{tabular}

TABLE V

SCHEDULE PARTITIONS WITH RESPECT TO FEASIBILITY FOR ELECTRIFICATION

\section{RESULTS FOR FLANDERS REGION}

Feathers statistics have been summarized in table IV. Results are presented in diagrams showing the power demand as function of time for the locations having respectively maximal energy requirement and power demand values over the complete day and during the specific tariff periods. It is interesting to note the ratio between peak power values for both scenarios. Even a simple random charging period allocation reduces peak power demand drastically. Diagram 2 shows the distribution function for accumulated daily car trip distances. One easily sees that at least $70 \%$ of the daily distances driven are less than the range of an electric vehicle (even after reduction for range anxiety and without recharging at work). Table $\mathrm{V}$ shows the fractions of feasible schedules determined in the second step (accounting for work location recharge). Note that only $10 \%$ (see CC_PROBABILITY in table III) of the schedules having a work trip have been assigned a CC (see section III-C). All power diagrams apply to scenarios where EV market penetration is $10 \%$ for nonworkers and 5\% for workers. Diagram 3 applies to the area with maximal power demand during low tariff period for scenario 1. Diagram 4 applies to the area with maximal power demand during regular tariff period for scenario 1. Diagram 5 applies to the area with maximal power demand during low tariff period for scenario 2. Diagram 6 applies to the area with maximal power demand during regular tariff period for scenario 2. Figure 7 projects the energy drawn from the 


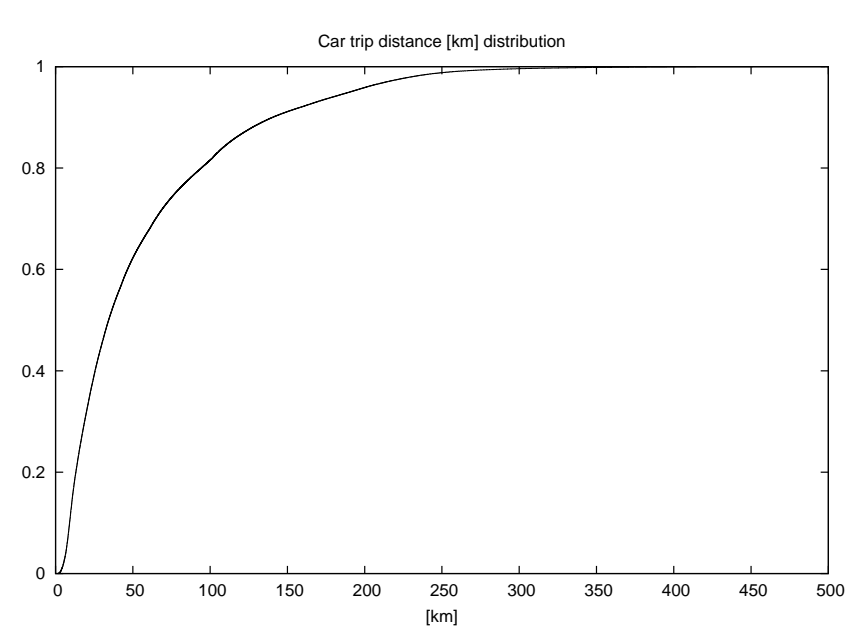

Fig. 2. Distribution function for average car trip distance

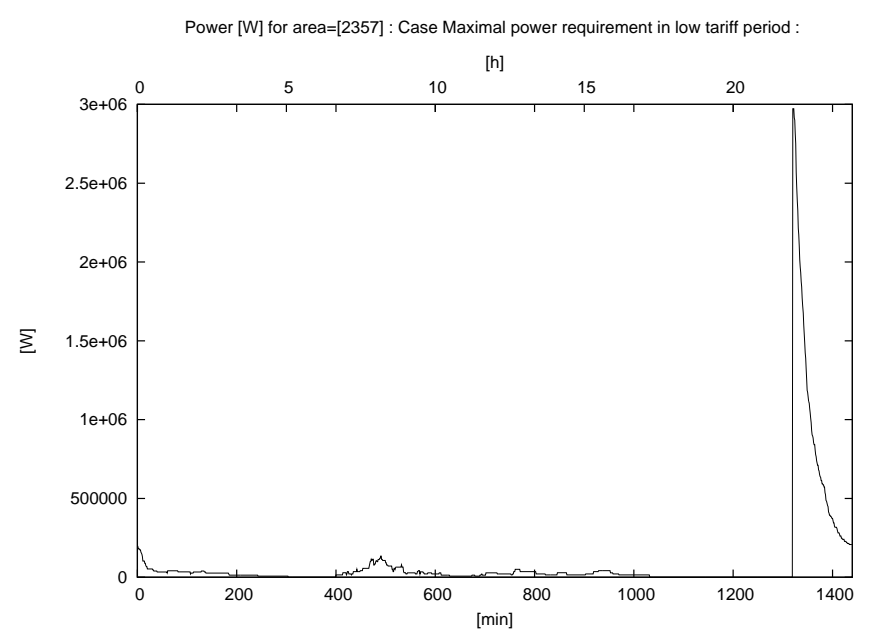

Fig. 3. Scenario 1 : Power demand for the zone having the biggest power demand peak during low tariff period

grid during the $24 \mathrm{~h}$ period considered. Figure 8 shows the peak power demand during low tariff hours for each zone for the same period. Darker shading on the maps corresponds to higher values.

\section{CONCLUSION}

Activity based transportation demand modelling uses census and survey data to model human behavior by microsimulation. It produces daily activity schedules for a synthetic population. It has been used to predict energy demand and power peaks due to electric vehicle charging as a function of time and location for several market penetration scenarios. Results have been calculated for the Flanders region : the power demand peak shaving effect of a simple charging strategy has been shown. Location specific predictions are available for smartgrid design. Daily overall trip distance distribution only provides a rough indication of the fraction of ICE vehicles that can be substituted by EV. On the other hand, AB-model microsimulation provides the data required for electrification feasibility calculations and smartgrid strategies evaluation.

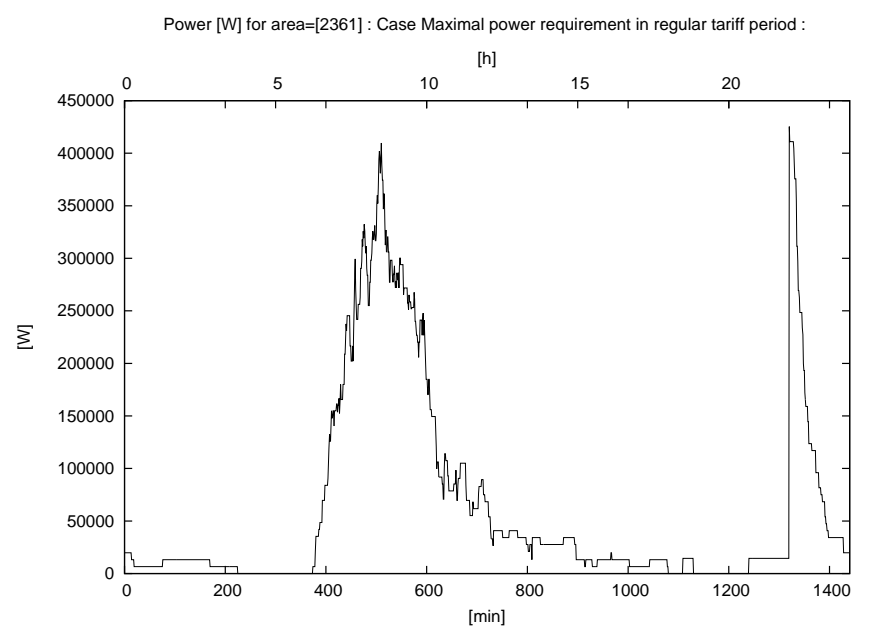

Fig. 4. Scenario 1 : Power demand for the zone having the biggest power demand peak during regular tariff period

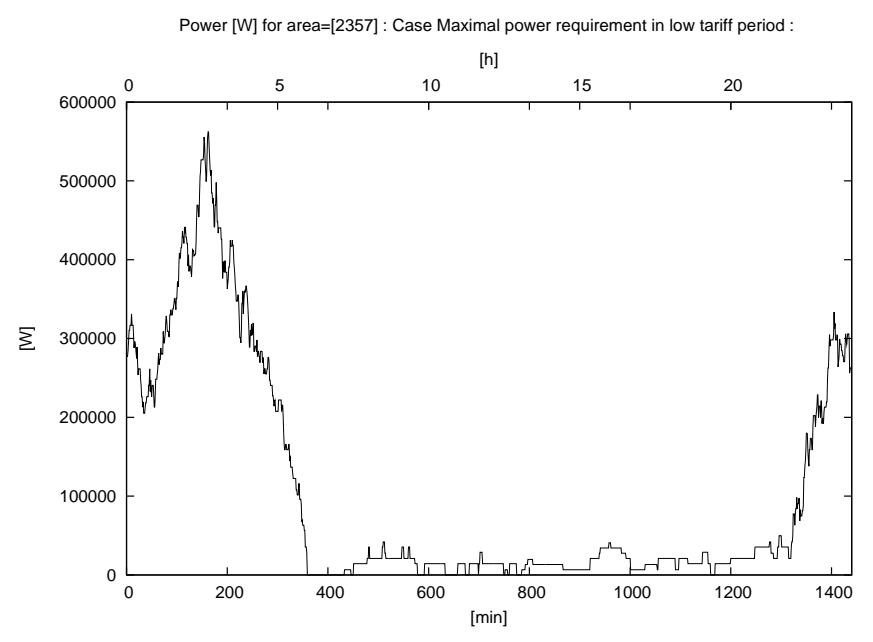

Fig. 5. Scenario 2 : Power demand for the zone having the biggest power demand peak during low tariff period

\section{FUTURE RESEARCH}

Although activity based models have a firm statistical basis, some aspects of reality do not yet have been translated to AB-model parameters. Therefore, this study shall be the base for two research paths. On one hand, more accurate technical and market related data need to be determined from literature, surveys and experimentation. Data about distance specific energy consumption in real situations are unreliable and need to be refined (cabin clima). The amount of car users who are able to charge at home has not been considered a limiting factor for the current study but could be one of the main factors when estimating EV market share.

On the other hand, more charging scenarios (e.g. using non contiguous periods) are to be investigated : the software allows for easy replacement of the charging strategy. A first software extension will remove the constraint of using a single vehicle for schedule trips executed by multi-car households.

Finally, AB-models and smartgrid models need to get inte- 


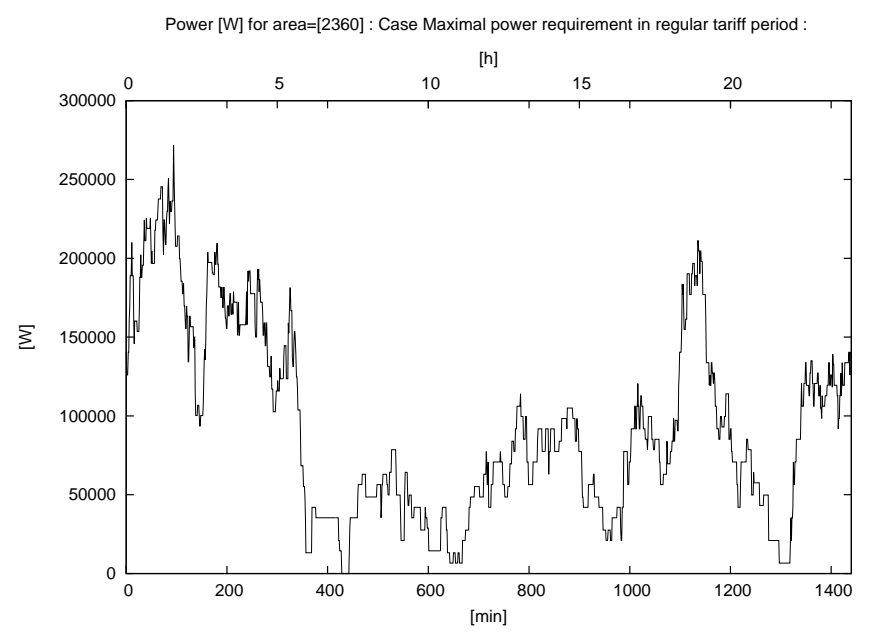

Fig. 6. Scenario 2 : Power demand for the zone having the biggest power demand peak during regular tariff period

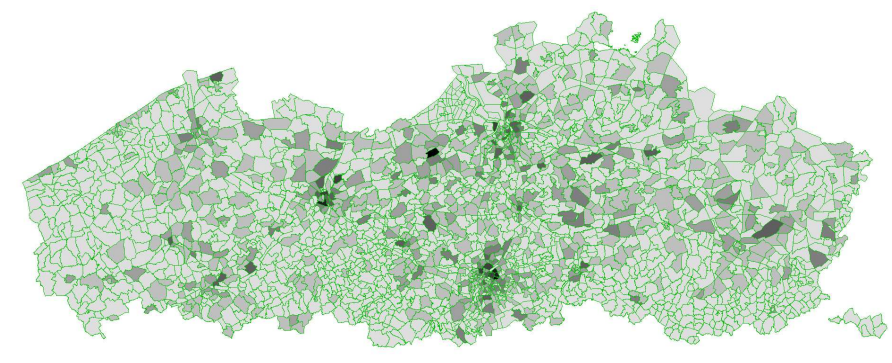

Fig. 7. Total energy requirement (EV only) over 24 hours : darker shading corresponds to higher value

grated in a closed loop. Since typical activity based models account for price elasticity and allow for learning, results feedback allows for evaluation of smartgrid strategies for charging timeslot allocation. Evaluation of the V2G concept requires integration of smartgrid controllers with $\mathrm{AB}$-models.

\section{REFERENCES}

[1] B. Kochan, T. Bellemans, M. Cools, D. Janssens, and G. Wets, "An estimation of total vehicle travel reduction in the case of telecommuting. detailed analyses using an activity-based modeling approach." Namur, Belgium, May 2011.

[2] A. P. M. D. PARQUE and B. CIUFFO, "Potential impact of electric vehicles on the electric supply system. a case study for the province of milan, italy," OPOCE, no. JRC53390, 2009. [Online]. Available: http://publications.jrc.ec.europa.eu/repository/handle/111111111/10593

[3] A. Perujo and B. Ciuffo, "The introduction of electric vehicles in the private fleet: Potential impact on the electric supply system and on the environment. a case study for the province of milan, italy," Energy Policy, vol. 38, no. 8, pp. 4549 - 4561, 2010. [Online]. Available: http://www.sciencedirect.com/science/article/B6V2W4YY8BPC-4/2/7486163b78fb3bb192edc3b8ce0807ea

[4] "Country pictures belgium," Apr. 2011. [Online]. Available: http://www.eci.ox.ac.uk/research/energy/

[5] "Energy consumption, $\mathrm{CO} 2$ emissions and other considerations related to battery electric vehicles," Oct. 2010.

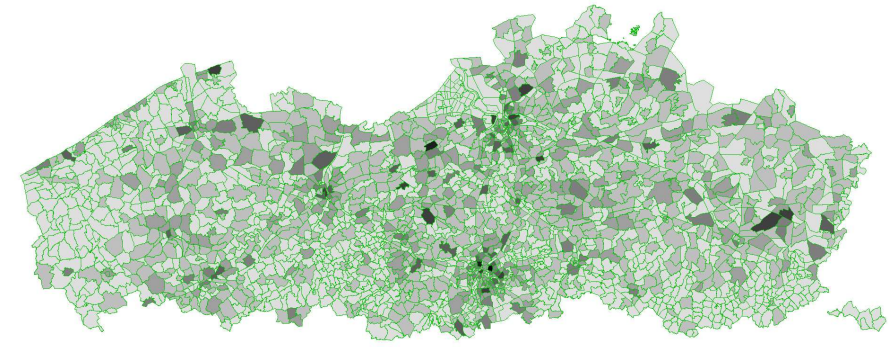

Fig. 8. Peak power demand (EV only) during low tariff hours : darker shading corresponds to higher value

[6] K. Clement-Nyns, E. Haesen, and J. Driesen, "Analysis of the impact of Plug-In hybrid electric vehicles on residential distri bution grids by using quadratic and dynamic programming," World Electric Vehicle Journal, vol. 3, 2009.

[7] R. A. Waraich and M. Galus, "Plug-in hybrid electric vehicles and smart grid: Investigations based on a Micro-Simulation," Dec. 2009.

[8] F. Nemry, G. Leduc, and M. Almudena, "Plug-in hybrid and BatteryElectric vehicles: State of the research and development and comparative analysis of energy and cost efficiency," 2009.

[9] "Transportdatabanken : Indicator PARC010," Tech. Rep., 2009. [Online]. Available: http://www.plan.be/

[10] E. Gutiérrez-i-Puigarnau and J. van Ommeren, "Welfare effects of distortionary company car taxation," Tinbergen Institute, Tinbergen Institute Discussion Papers 07-060/3, Aug. 2007. [Online]. Available: http://ideas.repec.org/p/dgr/uvatin/20070060.html

[11] "Recordaantal bedrijfswagens in belgië," Trends, 2007. [Online]. Available: http://trends.rnews.be/nl/economie/nieuws/finance/recordaantalbedrijfswagens-in-belgie/article-1194633700392.htm

[12] E. Cornelis, A. Malchair, T. Asperges, and K. Ramaekers, "COCA : Company cars analysis (Rapport final)," 2007. [Online]. Available: www.belspo.be/belspo/home/publ/pub_ostc/AP/rAP13_fr.pdf

[13] T. Bellemans, B. Kochan, D. Janssens, G. Wets, T. Arentze, and H. Timmermans, "Implementation framework and development trajectory of FEATHERS Activity-Based simulation platform," Transportation Research Record: Journal of the Transportation Research Board, vol. Volume 2175, no. Volume 2175 / 2010, pp. 111-119, Dec. 2010. [Online]. Available: http://trb.metapress.com/content/e5018jp286371r46/

[14] D. Wu, D. Aliprantis, and K. Gkritza, "Electric energy and power consumption by Light-Duty Plug-In electric vehicles," 2010.

[15] M. Kromer and J. Heywood, "Electric powertrains: Opportunities and challenges in the U.S. Light-Duty vehicle fleet," May 2007. 